Article

\title{
Fermentative Production of the Diamine Putrescine: System Metabolic Engineering of Corynebacterium Glutamicum
}

\author{
Anh Q. D. Nguyen, Jens Schneider ${ }^{\dagger}$, Gajendar Komati Reddy and Volker F. Wendisch * \\ Chair of Genetics of Prokaryotes, Faculty of Biology \& CeBiTec, Bielefeld University, \\ Universitätsstr. 25, 33615 Bielefeld, Germany; E-Mails: anguyen@cebitec.uni-bielefeld.de (A.Q.D.N.); \\ jens.schneider@evonik.com (J.S.); gkomati@CeBiTec.Uni-Bielefeld.de (G.K.R.) \\ $\dagger$ Current Address: Evonik Industries AG, Kantstraße 2, 33790 Halle/Westfalia, Germany. \\ * Author to whom correspondence should be addressed; E-Mail: volker.wendisch@uni-bielefeld.de; \\ Tel.: +49-521-106-5611; Fax: +49-521-106-5626.
}

Academic Editor: Dirk Steinhauser

Received: 26 February 2015 / Accepted: 13 April 2015 / Published: 24 April 2015

\begin{abstract}
Corynebacterium glutamicum shows great potential for the production of the glutamate-derived diamine putrescine, a monomeric compound of polyamides. A genome-scale stoichiometric model of a C. glutamicum strain with reduced ornithine transcarbamoylase activity, derepressed arginine biosynthesis, and an anabolic plasmid-addiction system for heterologous expression of $E$. coli ornithine decarboxylase gene speC was investigated by flux balance analysis with respect to its putrescine production potential. Based on these simulations, enhancing glycolysis and anaplerosis by plasmid-borne overexpression of the genes for glyceraldehyde 3-phosphate dehydrogenase and pyruvate carboxylase as well as reducing 2-oxoglutarate dehydrogenase activity were chosen as targets for metabolic engineering. Changing the translational start codon of the chromosomal gene for 2-oxoglutarate dehydrogenase subunit E1o to the less preferred TTG and changing threonine 15 of OdhI to alanine reduced 2-oxoglutarate dehydrogenase activity about five fold and improved putrescine titers by $28 \%$. Additional engineering steps improved further putrescine production with the largest contributions from preventing the formation of the by-product $\mathrm{N}$-acetylputrescine by deletion of spermi(di)ne $\mathrm{N}$-acetyltransferase gene snaA and from overexpression of the gene for a feedback-resistant $\mathrm{N}$-acetylglutamate kinase variant. The resulting C. glutamicum strain NA6 obtained by systems metabolic engineering accumulated
\end{abstract}


two fold more putrescine than the base strain, i.e., $58.1 \pm 0.2 \mathrm{mM}$, and showed a specific productivity of $0.045 \mathrm{~g} \cdot \mathrm{g}^{-1} \cdot \mathrm{h}^{-1}$ and a yield on glucose of $0.26 \mathrm{~g} \cdot \mathrm{g}^{-1}$.

Keywords: diamine production; putrescine; Corynebacterium glutamicum; 2-oxoglutatarate dehydrogenase; pyruvate carboxylase; glyceraldehyde 3-phosphate dehydrogenase; OdhI; spermidine $\mathrm{N}$-acetyltransferase; CgmR; $\mathrm{N}$-acetylglutamate kinase; gamma-glutamate kinase; genome-scale metabolic model; flux balance analysis

\section{Introduction}

In 2012, the total world market for polyamides was US \$22 billion, and with the increasing growth rate annually, it is expected to reach a market value of US \$27 billion by 2018 [1]. Due to their extreme durability and strength, polyamides are used in many applications from textiles, automotives, carpets, sportswear, to oil and gas industry [2,3]. An increasing demand for "green" polyamides is mostly driven by the rising consumer awareness concerning sustainability issues. Polymer precursors can be divided into three major groups: (i) monomers with terminal vinyl groups in polymers such as polystyrene, polyethylene (PE), polyvinylchloride (PVC), etc.; (ii) bifunctional monomers with terminal hydroxy, amino, and carboxy functionalities in polymers such as polyesters and polyamides; (iii) diisocyanates in polymers such as polyurethanes. In contrast to isocyanate and vinyl groups, which are rarely compatible with biological systems, the hydroxyl, carboxyl, and amino functional groups of polyamides and polyesters occur throughout the biological world, and their total synthesis by either biocatalysis or fermentation appears feasible [1].

A large number of the polyamide monomers can be produced, in principle, by bio-based routes, which led to the availability of a variety of different polyamides with excellent properties. Successful fermentative production of 1, 4-diaminobutane (putrescine) has recently been demonstrated [4,5]. So far, putrescine has been produced using engineered E. coli [6] and C. glutamicum [5]. The C. glutamicum system was developed further with the highest putrescine yield reported in bacteria $0.26 \mathrm{~g} \cdot \mathrm{g}^{-1}$ [4]. Tuning expression of ornithine transcarbamoylase gene $\arg F$ over 1000 fold through modulation of transcription and translation efficiencies was the key to balance low-level ornithine transcarbamoylase activity for obtaining high productivity and titer [4]. Putrescine was also produced from alternative carbon sources such as crude glycerol [7], hemicellulosic hydrolysates [8], amino sugars [9], thick juice [10] and by a biotin-prototrophic putrescine producing strain [11].

In this study, a genome-scale stoichiometric model of $C$. glutamicum was investigated by flux balance analysis with respect to the metabolic potential for putrescine production. Subsequently, putrescine production was optimized by engineering glycolysis, anaplerosis, 2-oxoglutarate dehydrogenase activity, proline biosynthesis, putrescine $\mathrm{N}$-acetylation and feedback control of arginine biosynthesis. 


\section{Results and Discussion}

\subsection{In Silico Characterization of Putrescine Production in C. glutamicum}

The optimum yield on glucose for $C$. glutamicum is $94 \%\left(\mathrm{~mol} \cdot \mathrm{mol}^{-1}\right)$ and, is $0.627 \mathrm{~mol}-\mathrm{C} \cdot \mathrm{mol}-\mathrm{C}^{-1}$ as calculated by flux balance analysis regardless of biomass formation (Table 1). Simulation results suggested that putrescine biosynthesis is constrained by the stoichiometry and furthermore by redox availability, indicated by a positive shadow price for NADH (not shown). Putrescine biosynthesis from carbon sources like lactate and acetate yielded less putrescine, but in contrast to glucose, the production was only constrained by stoichiometry. The putrescine yield on a more reduced carbon source like glycerol was higher $\left(0.653 \mathrm{~mol}-\mathrm{C} \cdot \mathrm{mol}^{-1} \mathrm{C}^{-1}\right)$ compared to glucose with the synthesis no longer being redox-constrained.

Table 1. Theoretical metabolic capacity of C. glutamicum for putrescine production with respect to different carbon sources. The carbon uptake was constrained to $24 \mathrm{mmol}-\mathrm{C}$ $\mathrm{gDCW}^{-1} \cdot \mathrm{h}^{-1}$. The degree of reduction $(\mathrm{K})$ and the yield of putrescine on the indicated carbon source $\mathrm{Y}_{\mathrm{P} / \mathrm{S}}$ are given.

\begin{tabular}{ccc}
\hline Carbon Source & $\mathbf{K}$ & $\mathbf{Y}_{\mathbf{P} / \mathbf{S}}\left[\mathbf{m o l}-\mathbf{C} \cdot \mathbf{m o l}^{-C^{-1}}\right.$ ] \\
\hline Glucose & 4.0 & 0.627 \\
Glycerol & 4.7 & 0.653 \\
Lactate & 4.0 & 0.511 \\
Acetate & 4.0 & 0.464 \\
\hline
\end{tabular}

The next step was to investigate the in silico flux distributions associated with different putrescine production rates (Figure 1). As shown in Figure 1A, the split ratio of carbon flux at the glucose-6phosphate node (Pgi/Zwf) without putrescine production was $72 \%$ to $21 \%$. This ratio differed slightly from the flux measured in the wild type and the flux calculated in simulation experiments by Shinfuku and colleagues, who determined ratios of $59 \%$ to $41 \%$ and $60 \%$ to $40 \%$, respectively [12]. When putrescine secretion had been increased stepwise up to $94 \%$, a flux redistribution was observed. The flux through the pentose phosphate pathway (PPP) increased up to $71 \%$ at $50 \%$ putrescine production with respect to the glucose uptake, indicating an increased NADPH demand for putrescine production (Figure 1B). Interestingly, if putrescine production increased even further, this did not lead to an increased flux through the PPP. Rather a decrease to $35 \%$ was observed at $94 \%$ putrescine flux. This decrease was compensated by an active malate enzyme (MalE) at a putrescine flux above 50\% (Figure 1B). MalE in combination with pyruvate carboxylase (Pyc) and malate dehydrogenase (Mdh) constitutes a transhydrogenase cycle [13], might supply NADPH for the reduction of glutamate to putrescine.

For biosynthesis of one mole putrescine from glucose three moles NADPH are consumed with one mole NADPH being formed by conversion of glucose to 2-oxoglutarate and three moles of NADPH being consumed by NADP-dependent glutamate dehydrogenase Gdh and NADP-dependent N-acetyl$\gamma$-glutamyl-phosphate reductase $\mathrm{ArgC}$ and indirectly by glutamate-dependent $\mathrm{N}$-acetyl-ornithine aminotransferase ArgD. Redox cofactor supply was not engineered in this work, but might prove relevant in further improving putrescine production by recombinant C. glutamicum. This may be particularly important when using less reduced carbon sources such as glycerol [7,14], D-gluconate, 
D-glucuronic acid (a component of plant xylans) or D-galacturonic acid (abundant in pectin-rich waste such as peels and pulps) [15]. Besides redox cofactor regeneration, carbon redirection from the tricarboxylic acid cycle (TCA) towards glutamate biosynthesis is crucial for high putrescine yields. The redirection may be achieved by reducing the 2-oxoglutarate dehydrogenase (OdhA) activity. The flux through 2-oxoglutarate dehydrogenase complex (ODHC) was $48 \%$ of wild-type conditions, when flux simulation was done with optimization towards growth. This flux became zero at putrescine yields of $0.75 \mathrm{~mol} \cdot \mathrm{mol}^{-1}$ or higher.

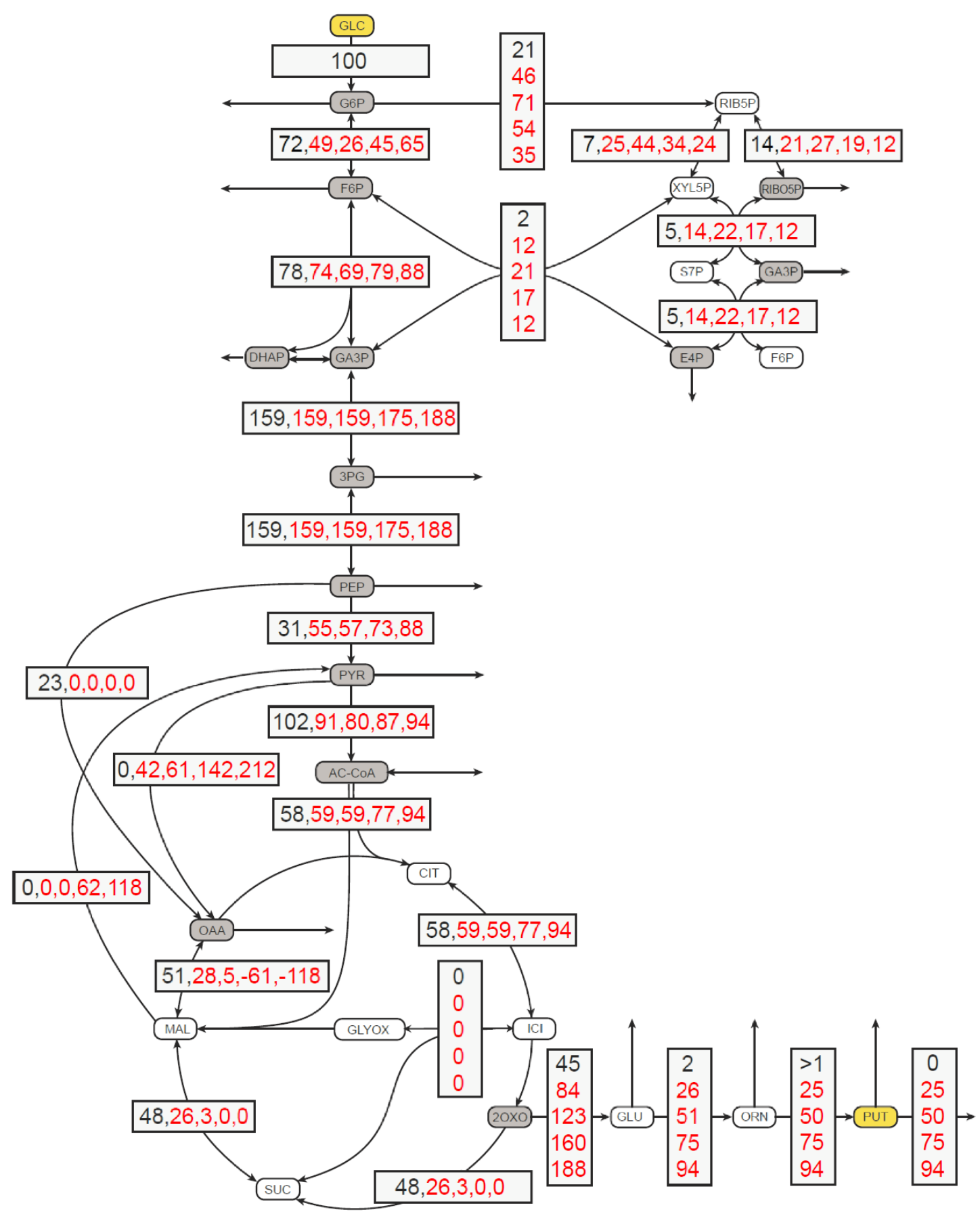

(A)

Figure 1. Cont. 


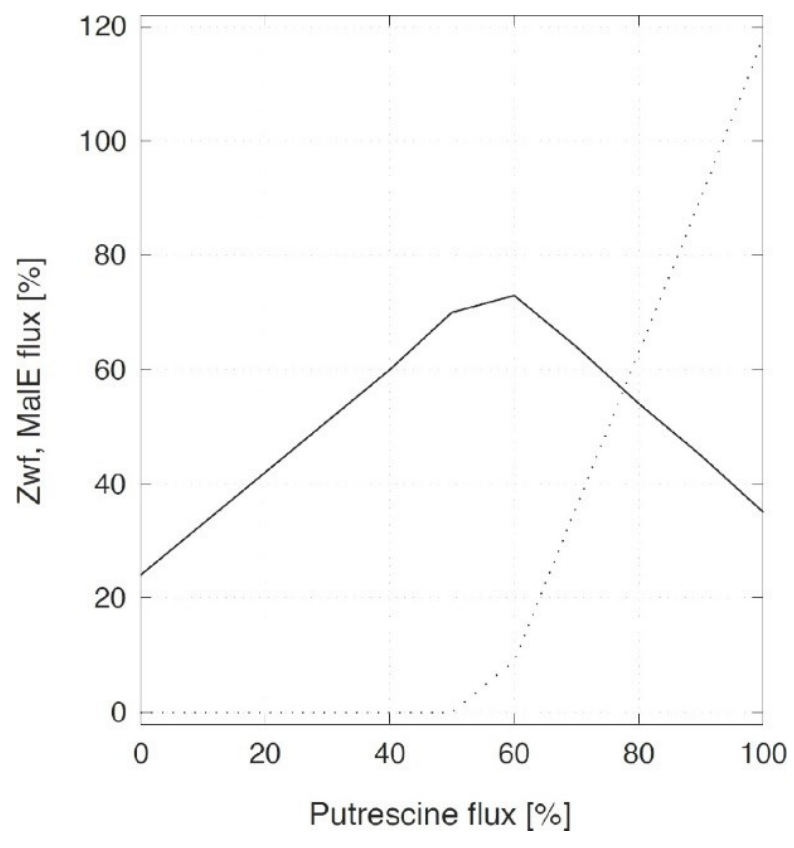

(B)

Figure 1. Metabolic flux distribution in C. glutamicum (A) and the relative flux through glucose 6-phosphate dehydrogenase Zwf and malate enzyme (MalE) (B) as a function of putrescine production. (A) Objective function was biomass flux, except for $100 \%$ putrescine flux. The metabolic flux was distributions were calculated in C. glutamicum without (in black) and with (in red) putrescine secretion to obtain yield coefficient (Y $/ \mathrm{s}$ ) of $25,50,75,94 \%$, respectively, relative to the glucose uptake rate. All fluxes are given in percent and are normalized to glucose uptake. Values are sorted by increasing putrescine flux. Solid line: Zwf flux, dotted line: MalE flux. For abbreviations: 1,3PG: 1,3-Bisphosphogylceric acid, 2OXO: 2-Oxoglutaric acid, 2PG: 2-Phosphoglyceric acid, 3PG: 3-Phosphoglyceric acid, AC-CoA: Acetyl-CoA, CIT: Citric acid, DHAP: Dihydroxyacetonephosphate, F6P: Fructose-6-phosphate, G6P: Glucose-6-phosphate, GA3P: Glyceraldyehyde-3-phosphate, GLC: Glucose, GLC-LAC: 6-Phosphogluconolactone, GLC6P: 6-Phosphogluconic acid, GLU: L-Glutamic acid, GLY: Glycerol, GLY3P: Glycerol-3phosphate, ICI: Isocitric acid, L-RIB5P: L-Ribulose-5-phosphate, MAL: Malic acid, NAC-GLU: N-Acteylglutamic acid, OAA: Oxalacetic acid, ORN: L-Ornithine, PEP: Phosphoenolpyruvic acid, PUT: Putrescine, PYR: Pyruvic acid, RIB: LRibulose, RIB5P: Ribulose-5-phosphate, RIBO5P: Ribose-5-phosphate, S7P: Sedoheptulose-7-phosphate, E4P: Erythrose-4-phosphate, SUC: Succinic acid. Arrows from intermediates marked in grey boxes perpendicular to the metabolic reactions indicate flux into biomass.

Next, the yields of the current producer strains [4] were compared with those predicted by flux balance analysis. The experimental yields matched the linear increase up to $0.21 \mathrm{~mol} \cdot \mathrm{mol}^{-1}$ (Table S1, Figure 2). The yield coefficients of the high yielding strains PUT21, PUT24 and PUT27 [4] were marginally lower than predicted for the network structure with constrained PPP and inactive MalE. Taken together, the simulations indicated that cofactor supply, carbon redirection or a combination of both might be limiting the putrescine yield in the current producer strains PUT21, PUT24 and PUT27. 


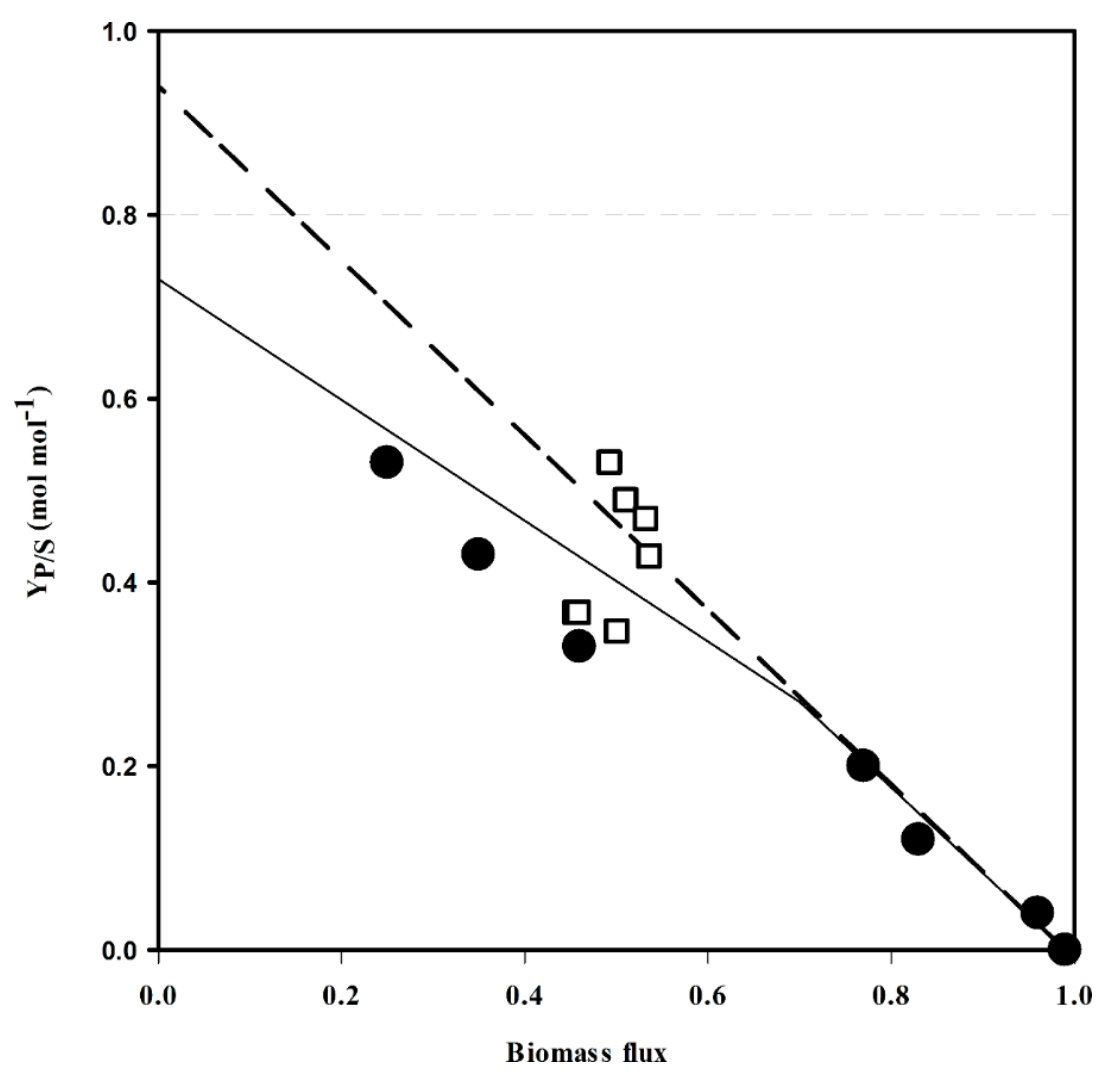

Figure 2. Comparison of theoretical and experimental putrescine yields. The putrescine flux response was analyzed by flux balance analysis with different biomass (the split ratio between Embden-Meyerhof-Parnas pathway (EMP) and pentose phosphate pathway (PPP) pathway was 6:4) when MalE was inactive (solid line) and active (dashed line). Circles: PUT3-27 [4], open squares: NA2-8.

\subsection{Reducing 2-Oxoglutarate Dehydrogenase Activity as a Target to Increase Putrescine Production}

2-Oxogluatarate is a branch point metabolite, which can be funneled either into the TCA cycle or glutamate biosynthesis. ODHC is a key enzyme in the TCA cycle consisting of three subunits: E1o is encoded by $o d h A$ [16], E2o is encoded by $a c e F$, and E3 is encoded by lpd [17]. Putrescine production should benefit from the reduction of ODHC activity as suggested by the flux simulation and two approaches were followed: decreasing synthesis of OdhA, a subunit of ODHC [16] and/or inhibiting ODHC by maintaining its binding with OdhI [18]. Reducing synthesis of OdhA by exchanging the translational start codon from GTG to TTG in the chromosome of strain PUT21 reduced ODHC activity from $11 \pm 2 \mathrm{mU} / \mathrm{mg}$ to $7 \pm 1 \mathrm{mU} / \mathrm{mg}$ (Figure 3) and increased putrescine production by $15 \%$ (Figure 3). Alternatively, maintaining inhibition of ODHC by exchanging threonine residues 14 or 15 or both of OdhI to alanine resulted in partial or complete loss of phosphorylation and inactivation of OdhI by PknG [18]. Strains PUT21odhI ${ }^{\mathrm{T} 14 \mathrm{~A}}$ and PUT21odhI $I^{\mathrm{T} 15 \mathrm{~A}}$ showed ODHC activities of $10 \pm 2$ and $7 \pm 1 \mathrm{mU} / \mathrm{mg}$, respectively (Figure 3) and produced $32 \pm 3 \mathrm{mM}$ and $32 \pm 2 \mathrm{mM}$ putrescine (Figure 3 ). Combining the chromosomal changes $o d h A^{\mathrm{TTG}}$ and $o d h I^{\mathrm{T} 15 \mathrm{~A}}$ yielded strain NA2, reduced ODHC activity to $2 \pm 0 \mathrm{mU} / \mathrm{mg}$ (Figure 3 ) and increased putrescine production by $28 \%(38.1 \pm 0.2 \mathrm{mM})$ as compared to PUT21 (29.2 $\pm 2.8 \mathrm{mM}$, Figure 3$)$. Significant concentrations $(>2 \mathrm{mM})$ of glutamate or other amino acids were not detected in culture broth at the end of all cultivations. 


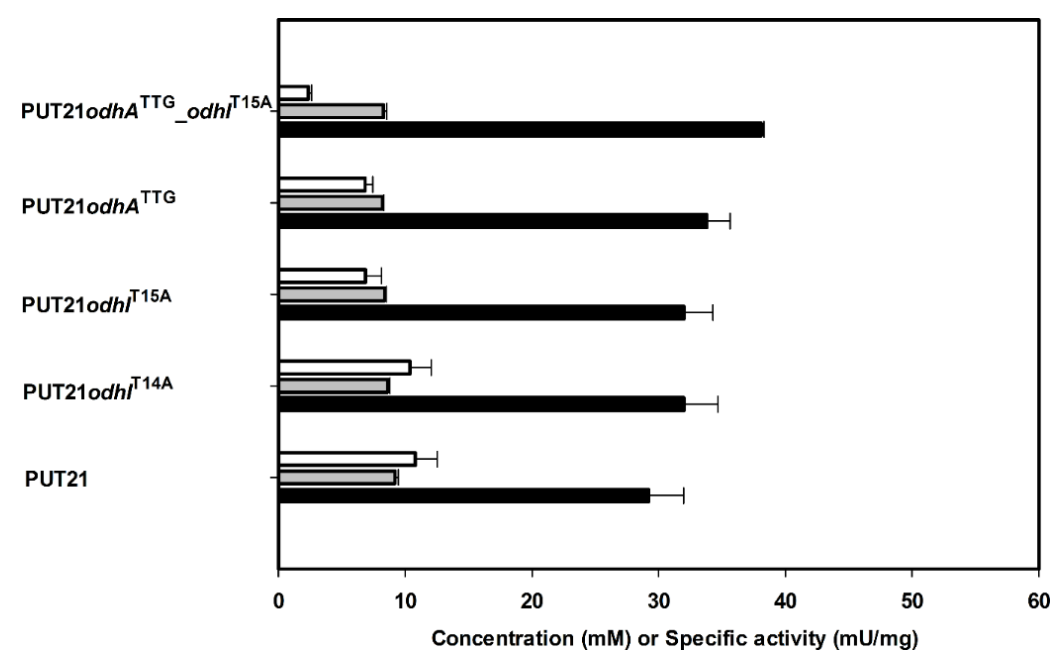

Figure 3. 2-Oxoglutarate dehydrogenase as a target to increase production of putrescine production. Concentrations of putrescine (black bar) and N-acetylputrescine (grey bar) in supernatants and 2-oxoglutarate dehydrogenase activities in crude extracts (white bar) of different strains are given as means and standard errors of three independent cultivations. Cells were grown in CGXII medium with $20 \mathrm{~g} \cdot \mathrm{L}^{-1}$ glucose and $1 \mathrm{mM}$ IPTG.

Table 2. Growth Rates and Putrescine Production Parameters Obtained with Various Engineered C. glutamicum Strains ${ }^{\text {a }}$.

\begin{tabular}{|c|c|c|c|c|c|c|}
\hline Strain & $\begin{array}{c}\text { Growth Rate } \\
\boldsymbol{\mu}^{\mathrm{b}}\left(\mathbf{h}^{-1}\right)\end{array}$ & $\begin{array}{c}\text { Putrescine } \\
\text { Accumulation (mM) }\end{array}$ & $\begin{array}{l}\text { Product Yield } \\
Y_{P / s^{b}}\left(g \cdot g^{-1}\right)\end{array}$ & $\begin{array}{c}\text { Biomass } \\
\text { Yield } \mathrm{Y}_{\mathrm{X} / \mathrm{s}^{\mathrm{b}}} \\
\left(\mathrm{g} \cdot \mathrm{g}^{-1}\right) \\
\end{array}$ & $\begin{array}{c}\text { Volumetric } \\
\text { Productivity } \\
\mathbf{Q}_{\mathrm{p}}{ }^{\mathrm{c}}\left(\mathbf{g} \cdot \mathbf{L}^{-1} \cdot \mathbf{h}^{-1}\right)\end{array}$ & $\begin{array}{c}\text { Specific } \\
\text { Productivity } \\
\mathbf{q}_{\mathbf{p}}{ }^{\mathbf{c}}\left(\mathbf{g}^{\cdot} \mathbf{g}^{-1} \cdot \mathbf{h}^{-1}\right)\end{array}$ \\
\hline PUT21 & 0.19 & $29.2 \pm 2.8$ & 0.13 & 0.28 & 0.11 & 0.020 \\
\hline PUT21 proB ${ }^{\mathrm{GTG}}$ & 0.22 & $31.2 \pm 3.0$ & 0.14 & 0.27 & 0.12 & 0.022 \\
\hline PUT21proB $B^{\mathrm{TTG}}$ & 0.14 & $46.1 \pm 2.8$ & 0.20 & 0.29 & 0.14 & 0.024 \\
\hline NA2 & 0.17 & $38.1 \pm 0.2$ & 0.17 & 0.25 & 0.14 & 0.028 \\
\hline NA3 & 0.16 & $41.9 \pm 1.1$ & 0.18 & 0.22 & 0.14 & 0.032 \\
\hline NA4 & 0.16 & $41.6 \pm 1.1$ & 0.18 & 0.26 & 0.14 & 0.027 \\
\hline NA5 & 0.15 & $48.3 \pm 3.3$ & 0.21 & 0.23 & 0.19 & 0.041 \\
\hline NA6 & 0.17 & $58.1 \pm 0.2$ & 0.26 & 0.23 & 0.21 & 0.045 \\
\hline NA7 & 0.20 & $53.4 \pm 2.2$ & 0.24 & 0.24 & 0.20 & 0.042 \\
\hline NA8 & 0.20 & $51.2 \pm 0.5$ & 0.23 & 0.24 & 0.20 & 0.041 \\
\hline
\end{tabular}

${ }^{a}$ Means of three independent cultivations in CGXII medium with $20 \mathrm{~g} \mathrm{~L}^{-1}$ glucose and $1 \mathrm{mM}$ IPTG are given.

${ }^{\mathrm{b}}$ Relative standard errors were $10 \%$ or less. ${ }^{\mathrm{c}}$ Relative standard errors were $15 \%$ or less.

Low or even absent ODHC activity has been associated with glutamate overproduction by C. glutamicum since long (see, e.g., [19]) and deletion of odhA led to overproduction of glutamate [20]. The importance of ODHC in C. glutamicum metabolism is reflected in its elegant activity control [21]. In its unphosphorylated form, the small protein OdhI inhibits ODHC activity [18] by direct interaction of its FHA domain with the E1o subunit of ODHC [22]. The protein kinases PknG, PknA, PknB and PknL phosphorylate OdhI at T14 and/or T15 [18,23,24]. Manipulation of OdhI phosphorylation by mutation of the threonine residues inOdhI or by deletion of the gene for protein kinase PknG has been shown previously to increase glutamate production [24]. In the present study, reducing translation of 
odhA due to changing the translational start codon GTG to the less preferred TTG and exchanging the threonine residue 15 of OdhI, which is phosphorylated by the protein kinases, were combined. As consequence, about a five fold reduced ODHC activity was observed (Figure 3), but growth hardly slowed (compare NA2 with PUT21 in Table 2) (growth rate of $0.16 \mathrm{~h}^{-1}$ ). This may explain why indications of genetic instability were not observed with the strains described here, whereas several different classes of suppressor mutants arose upon deletion of odhA [20] and, thus, reducing ODHC activity may be superior to its complete absence.

\subsection{Increasing Precursor Supply Flux for Putrescine Production}

In order to increase the supply of 2-oxoglutarate as precursor for putrescine biosynthesis, two targets were chosen: glyceraldehyde 3-phosphate dehydrogenase in glycolysis and pyruvate carboxylase for anaplerosis of the TCA cycle. Since glyceraldehyde 3-phosphate dehydrogenase has been shown to be limiting glycolytic flux at least under oxygen-deprivation conditions [25], overexpression of its gene gapA in PUT21 was tested. As compared to PUT21 carrying the empty vector, PUT21 overexpressing gapA produced $39 \%$ more putrescine $(44.0 \pm 2.0 \mathrm{mM}$ as compared to $29.5 \pm 1.0 \mathrm{mM}$, Figure 4).

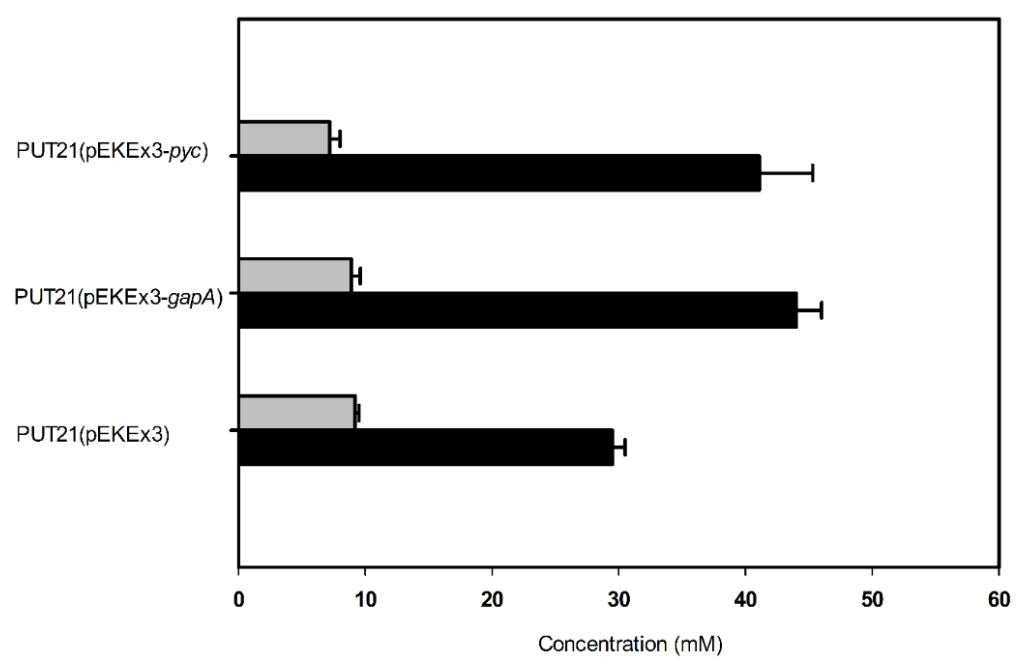

Figure 4. Effect of pEKEx3-based overexpression of gapA and $p y c$ in PUT21 on the production of putrescine (black bar) and $\mathrm{N}$-acetylputrescine byproduct (grey bar). Cells were grown in CGXII medium with $20 \mathrm{~g} \cdot \mathrm{L}^{-1}$ glucose and $1 \mathrm{mM}$ isopropyl $\beta$-D-1thiogalactopyranoside (IPTG). Means and standard error of three independent cultivations are shown.

Since pyruvate carboxylase is the major anaplerotic enzyme in C. glutamicum [26] and overexpression of its gene improved glutamate and lysine production [27], pyc was overexpressed in PUT21. C. glutamicum PUT21(pEKEx3-pyc) produced $41 \pm 4 \mathrm{mM}$ putrescine, an increase by $39 \%$ as compared to the empty vector carrying control strain ( $29 \pm 1 \mathrm{mM}$, Figure 4).

Overexpression of $p y c$ and gapA improved putrescine accumulation (Figure 4), but the individual effects were not additive (compare NA3 and NA4 in Table 2 with Figure 4). Whereas overexpression of $p y c$ was expected to increase anaplerosis and putrescine yield as reported previously for lysine and 
glutamate production [27], overexpression of gapA was expected to accelerate glucose utilization [28] and in consequence productivity. The simulation only showed a small flux increase from glyceraldehyde 3-phosphate to 3-phosphoglycerate in response to an increased theoretical putrescine yield, which is consistent with metabolic flux analysis data reported previously for glutamate overproduction in C. glutamicum [29]. Overexpression of gapA from plasmid pVWEx1 did neither change the growth rate nor the volumetric productivity, but improved the specific productivity due to reduced biomass formation (compare NA2 and NA3 in Table 2). It is not clear if gapA overexpression led to faster glucose consumption. The positive effect of gapA overexpression on putrescine accumulation varied with the expression plasmid used. Based on plasmid pVWEx1, GAPDH activity increased to $160 \pm 10$ $\mathrm{mU} / \mathrm{mg}$, while plasmid pEKEx3 led $260 \pm 10 \mathrm{mU} / \mathrm{mg}$ [30]. This might explain the relatively small effect observed in C. glutamicum NA3.

\subsection{Decreasing Conversion of Glutamate to Proline}

As putrescine production competes with proline biosynthesis for glutamate, it was tested if reducing proline biosynthesis positively affects putrescine production. To this end, the translational start codon of proB encoding $\gamma$-glutamate kinase, the initial enzyme of proline biosynthesis, was exchanged from ATG to GTG and TTG in the chromosome of strain C. glutamicum PUT21 by replacement mutagenesis. Indeed, putrescine production increased when the $p r o B$ gene contained the less preferred translational start codons GTG and TTG (Figure 5). Notably, PUT21 proB ${ }^{\mathrm{TTG}}$ did not produce N-acetylputrescine as a side product and $46.1 \pm 2.8 \mathrm{mM}$ putrescine accumulated. Thus, putrescine production benefitted from reducing entry of glutamate into proline biosynthesis.

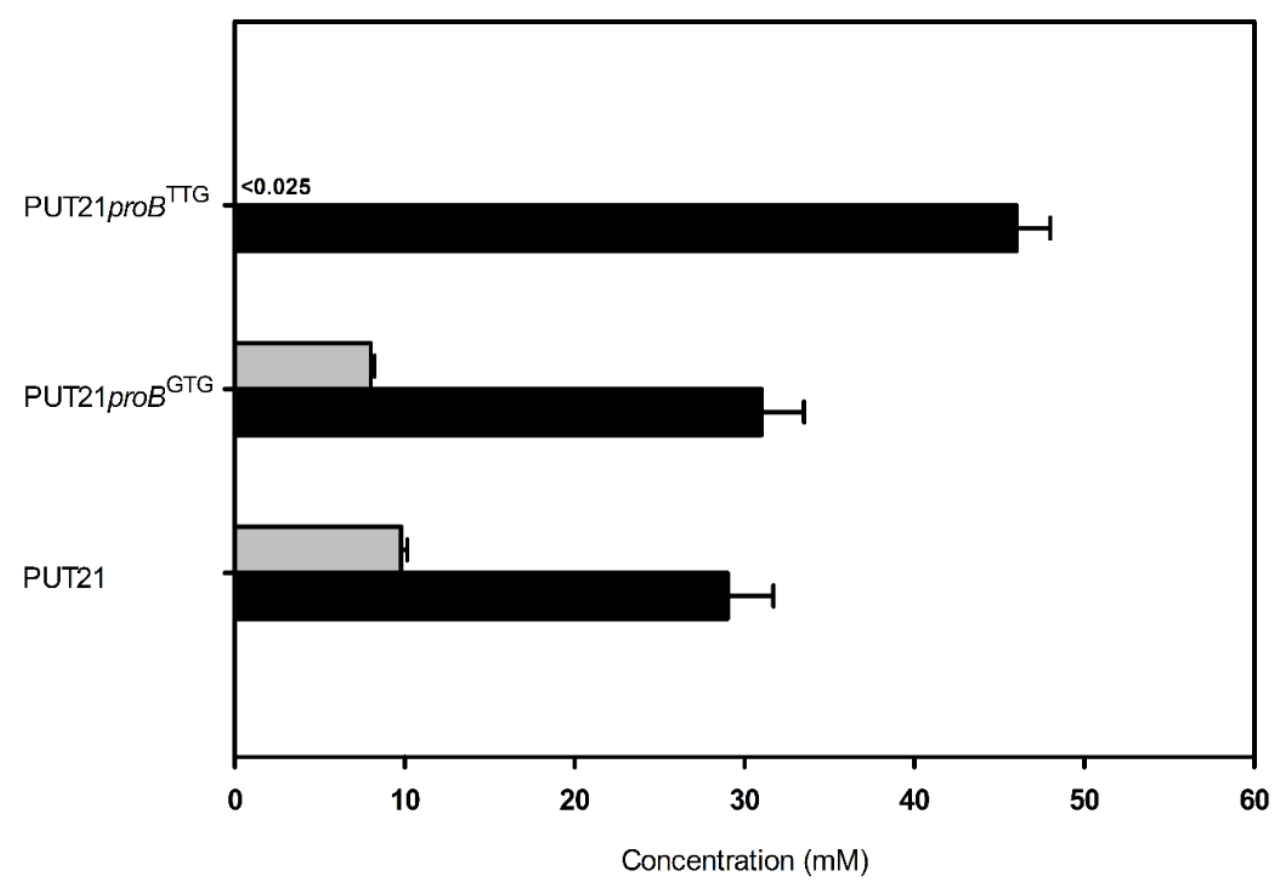

Figure 5. Putrescine production (black bar) and N-acetylputrescine byproduct (grey bar) in PUT21-derived strains carrying $\operatorname{pro} B$ with different translational start codons. Cells were grown in CGXII medium with $20 \mathrm{~g} \cdot \mathrm{L}^{-1}$ glucose and plasmid encoded genes were induced with $1 \mathrm{mM}$ IPTG. Means and standard error of three independent experiments are shown. 
Similarly, deletion of $\operatorname{proB}$ has been shown to improve ornithine production [31,32], however, as a consequence the resulting strains require either complex media components or addition of proline as a supplement to the culture medium. Interestingly, strain PUT21 proB ${ }^{\mathrm{TTG}}$ produced more putrescine, but no $\mathrm{N}$-acetylputrescine accumulated (Figure 5), although snaA, the gene for spermi(di)ne N-acetyltransferase, was intact. This effect is currently not understood, but activity or synthesis of spermi(di)ne $\mathrm{N}$-acetyltransferase may be reduced in PUT21 rroB $^{\mathrm{TTG}}$ due to higher levels of glutamate or lower levels of proline or intermediates of proline biosynthesis. Proline is known to affect binding of ArgR to the $\arg B$ promoter region [33], but this does not pertain to the putrescine strains described here because $\arg R$ is deleted in all of them. Proline is the major compatible solute of $C$. glutamicum and is produced in response to high osmolality [34] or taken up into the cell by osmo-regulated EctP and ProP or for anabolic purposes by PutP [35]. It is currently unknown if snaA expression or SnaA activity is osmo-regulated. Nonetheless, the effect of $\operatorname{proB}^{\mathrm{TTG}}$ on abrogating $\mathrm{N}$-acetylputrescine as a side product improved putrescine titers.

\subsection{Combinatorial Construction of Putrescine Overproducing C. glutamicum Strains}

Strain improvement was based on C. glutamicum PUT21, which produced $29.2 \pm 2.8 \mathrm{mM}$ putrescine from $20 \mathrm{~g} \cdot \mathrm{L}^{-1}$ glucose (Table 2). Strain NA2 carried the chromosomal changes $o d h A^{\mathrm{TTG}}$ and $o d h I^{\mathrm{T} 15 \mathrm{~A}}$ and produced $38.1 \pm 0.2 \mathrm{mM}$ (Table 2). Overexpression of gapA in NA2 yielded strain NA3, which showed increased glyceraldehyde 3-phosphate dehydrogenase activity (160 \pm 10 as compared $130 \pm 10 \mathrm{mU} / \mathrm{mg}$ ) and produced $41.9 \pm 1.1 \mathrm{mM}$ putrescine (Table 2, Figure 6). Additional overexpression of pyc did not increase putrescine production by strain NA4 (Figure 6). However, NA5, which showed increased feedback-resistant $\mathrm{N}$-acetyl glutamate kinase activity due to additional overexpression of $\arg B^{\mathrm{A} 49 \mathrm{~V} / \mathrm{M} 54 \mathrm{~V}}$ [36], produced more putrescine, namely $48.3 \pm 3.3 \mathrm{mM}$ (Figure 6). Overexpression of $\arg B^{\mathrm{A} 49 \mathrm{~V} / \mathrm{M} 54 \mathrm{~V}}$ improved putrescine overproduction although arginine accumulation was avoided in all putrescine producing strains described in this study due to leaky expression of $\arg F$. Thus, it is possible that overexpression of genes for various other feedback-alleviated N-acetylglutamate kinase variants [37,38] may increase putrescine production further. Although all $\mathrm{N}$-acetylglutamate kinase variants have been selected for loss of feedback inhibition by arginine, it is conceivable that $\mathrm{N}$ acetylglutamate kinase is inhibited by putrescine, ornithine or other arginine biosynthesis intermediates and that some of the described variants may have lost this putative feedback inhibition as well.

Recently, N-acetylation of putrescine was identified and the responsible protein SnaA characterized as spermi(di)ne N-acetyltransferase [39]. Deletion of snaA in C. glutamicum NA5 abrogated formation of $\mathrm{N}$-acetylputrescine as a side-product and $58.1 \pm 0.2 \mathrm{mM}$ putrescine accumulated (Figure 6). Since the TetR-family transcriptional repressor CgmR represses cgmAR expression in dependence of diamines [39,40] and since overexpression of the putative export permease gene $\operatorname{cgmA}$ improved putrescine and cadaverine production [39,41], the repressor gene $c g m R$ was deleted in C. glutamicum NA6 to yield strain NA7. However, putrescine production by strain NA7 was not improved (53.4 \pm 2.2 , Table 2), but slightly reduced as compared to strain NA6 (Figure 6). The translational start codon exchange from ATG to TTG of the chromosomal proline biosynthesis gene proB in C. glutamicum NA7 yielded strain NA8, which produced less putrescine $(51.2 \pm 0.5 \mathrm{mM})$ than strain C. glutamicum NA6 (Figure 6). 


\section{Strains Genotype}

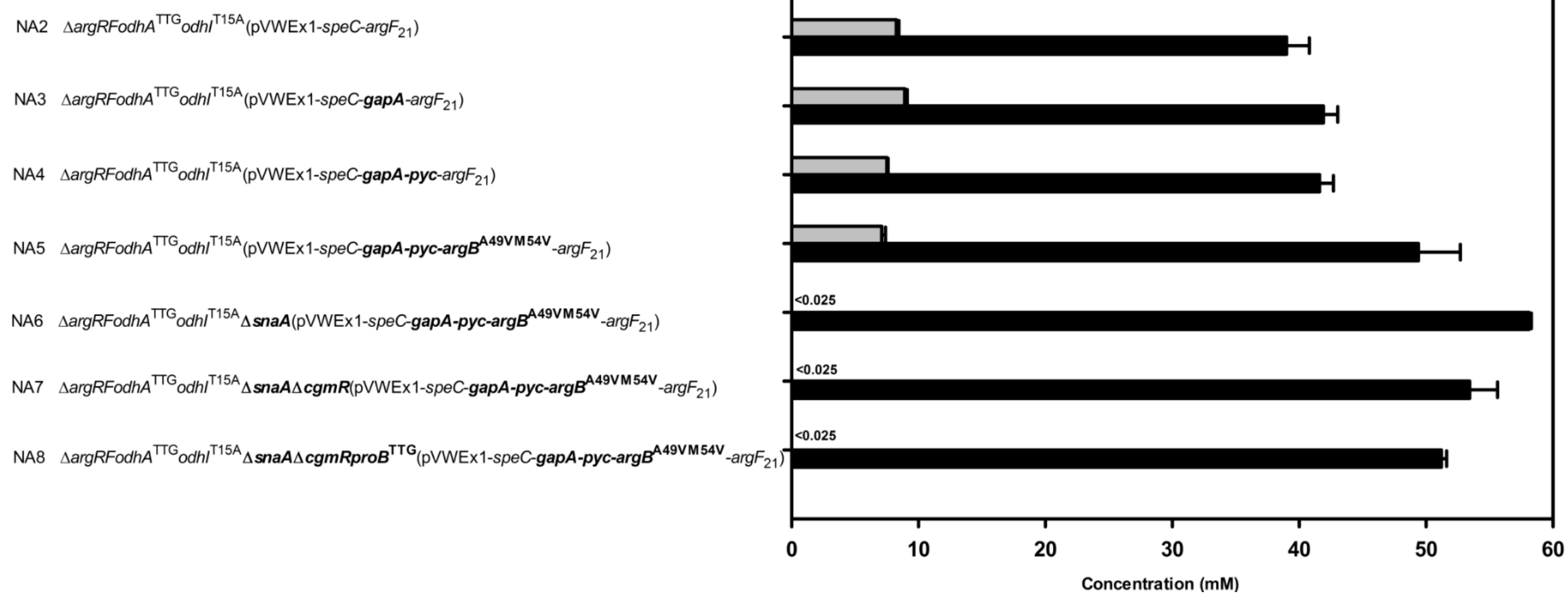

Figure 6. Effect of deletion and overexpression of engineering target genes in C. glutamicum strain NA2 on the production of putrescine (black bars) and $\mathrm{N}$-acetylputrescine (grey bars). Genetic changes introduced to the chromosome of C. glutamicum NA2 and to plasmid pVWEx1-speC- $\arg F_{21}$ are highlighted in bold. Genes for feedback-resistant N-acetylglutamate kinase ( $\left.\arg B^{\mathrm{A} 49 \mathrm{~V} / \mathrm{M} 54 \mathrm{~V}}\right)$, pyruvate carboxylase (pyc) and glyceraldehyde 3-phosphate dehydrogenase (gapA) were added to plasmid pVWEX1-speC-argF $F_{21}$, the translational start codon exchange of the $\gamma$-glutamate kinase gene $\operatorname{proB}$ from ATG to TTG was introduced in the chromosome, while the spermi(di)ne N-acetyltransferase gene snaA and the regulatory gene $c g m R$ were deleted. Cells were grown in CGXII medium with $20 \mathrm{~g} \cdot \mathrm{L}^{-1} \mathrm{glucose}$ and $1 \mathrm{mM}$ IPTG. Means and standard errors of three independent cultivations are shown. 
Deletion of snaA was beneficial for improving putrescine production by abrogating formation of $\mathrm{N}$-acetylputrescine as by-product (Figure 6) as observed previously. Since all strains lacking SnaA (NA6, NA7, NA8 in Figure 6 and strains described in [39]) did not accumulate N-acetylputrescine, other $\mathrm{N}$-acetyltransferases including ornithine $\mathrm{N}$-acetyltransferase ArgJ, which is overexpressed in the putrescine producing strains, did not compensate for the lack of SnaA. SnaA acetylates a number of diamines and triamines including cadaverine using acetyl-CoA or propionyl-CoA as donors [39] and deletion of snaA also improved production of the diamine cadaverine by abrogating formation of $\mathrm{N}$-acetylcadaverine as by-product [42]. Overexpression of putative putrescine permease gene $\operatorname{cgm} A$ or deletion of $\operatorname{cgmAR}$ repressor CgmR improved putrescine formation [39], but not in a $\triangle$ snaA background as observed here (compare NA6 and NA7 in Figure 6) and previously [39]. However, it is not known if $\mathrm{N}$-acetylputrescine affects $\mathrm{CgmA}$ or $\mathrm{CgmR}$ or if the higher putrescine concentrations in strains lacking SnaA are sufficient to trigger maximal putrescine export.

Production results from strains NA2 to NA8 were compared with data predicted by flux balance analysis (Table S1, Figure 2). The yield coefficients of strains NA2, NA3 and NA4 were slightly lower than predicted with constrained PPP and inactive MalE. However, data obtained for strains NA5 to NA8 fitted the prediction when MalE is active. Further analysis needs to be done to confirm the role of MalE in these strains.

Taken together, combinatorial strain development led to the efficient putrescine producer C. glutamicum NA6, which accumulated $58.1 \pm 0.2 \mathrm{mM}$ putrescine with a yield on glucose of 0.26 $\mathrm{g} \cdot \mathrm{g}^{-1}$ and a volumetric productivity of $0.21 \mathrm{~g} \cdot \mathrm{L}^{-1} \cdot \mathrm{h}^{-1}$. As shown previously, product titers can be increased in fed-batch processes, e.g., up to $211 \mathrm{mM}$ [4]. Strain NA6 showed the same product yield as PUT24 [4], however, the maximal putrescine titer was reached twice faster. The highest specific productivity of $0.75 \mathrm{~g} \cdot \mathrm{L}^{-1} \cdot \mathrm{h}^{-1}$ has been described for $E$. coli in a fed-batch process [6]. Since this $E$. coli strain showed a yield of $0.168 \mathrm{~g} \cdot \mathrm{g}^{-1}$ [6], while $C$. glutamicum NA6 showed a yield of $0.26 \mathrm{~g} \cdot \mathrm{g}^{-1}$, it appears possible to achieve similar or even higher specific productivities than with the $E$. coli strain.

These putrescine production capability was due to improved precursor supply (overexpression of gapA and $p y c$; reduced ODHC activity as consequence of $o d h A^{\mathrm{TTG}}$ and $o d h I^{\mathrm{T} 15 \mathrm{~A}}$, reduced ornithine transcarbamoylase activity due plasmid-borne expression of $\arg F_{21}$ and chromosomal $\arg F$ deletion), abrogated $\mathrm{N}$-acetylation of putrescine (snaA deletion), derepressed arginine biosynthesis ( $\arg R$ deletion) and feedback-resistant $\mathrm{N}$-acetylglutamate kinase $\left(\arg B^{\mathrm{A} 49 \mathrm{~V} / \mathrm{M} 54 \mathrm{~V}}\right)$, and heterologous expression of E. coli ornithine decarboxylase gene speC via an anabolic plasmid-addiction system.

\section{Experimental Section}

\subsection{Bacterial Strains and Culture Conditions}

Strains and plasmids used in the present work are listed in Table 3. C. glutamicum and E. coli strains were routinely grown in lysogeny broth (LB) $\left(10 \mathrm{~g} \cdot \mathrm{L}^{-1}\right.$ tryptone, $5 \mathrm{~g} \cdot \mathrm{L}^{-1}$ yeast extract, $10 \mathrm{~g} \cdot \mathrm{L}^{-1}$ sodium chloride) in $500 \mathrm{~mL}$ baffled flasks on a rotary shaker $(120 \mathrm{rpm})$ at $30^{\circ} \mathrm{C}$ or $37^{\circ} \mathrm{C}$. Briefly, a $50 \mathrm{~mL}$ LB seed culture of $C$. glutamicum was inoculated from an agar plate and grown overnight. The cells were harvested by centrifugation (4000 $\times \mathrm{g}, 10 \mathrm{~min})$ and washed once with CGXII minimal medium [43] lacking the carbon source. Subsequently, $50 \mathrm{~mL}$ CGXII medium, containing $20 \mathrm{~g} \cdot \mathrm{L}^{-1}$ glucose and 
necessary supplements, was inoculated to an initial optical density at $600 \mathrm{~nm}$ of 0.5 . Growth was followed by measuring the optical density at $600 \mathrm{~nm}$. The biomass concentration was calculated from $\mathrm{OD}_{600}$ values using an experimentally determined correlation factor of $0.25 \mathrm{~g}$ cell dry weight (DW) $\mathrm{L}^{-1}$ for $\mathrm{OD}_{600}$ of 1 . When necessary, the growth medium was supplemented with kanamycin $\left(25 \mu \mathrm{g} \cdot \mathrm{mL}^{-1}\right)$, spectinomycin $\left(100 \mu \mathrm{g} \cdot \mathrm{mL}^{-1}\right)$, and isopropyl $\beta$-D-1-thiogalactopyranoside (IPTG) $(1 \mathrm{mM})$.

Table 3. C. glutamicum Strains and Plasmids.

\begin{tabular}{|c|c|c|}
\hline Name & Relevant Genotype/Information & Refs. \\
\hline \multicolumn{3}{|l|}{$\underline{\text { Strains }}$} \\
\hline ORN1 & $\begin{array}{l}\text { In-frame deletion of } \arg R \text { and } \arg F, \mathrm{~L} \text {-ornithine overproducing strain derived from } \\
\text { C. glutamicum ATCC13032; auxotrophic for L-arginine }\end{array}$ & [5] \\
\hline PUT21 & ORN1 carrying plasmid pVWEx1-speC-argF $F_{21}$ & [4] \\
\hline PUT21odh $A^{\text {TTG }}$ & $\begin{array}{l}\text { PUT21 with replacement of translational start codon GTG of } \\
\text { chromosomal } o d h A \text { of by TTG }\end{array}$ & This study \\
\hline PUT21odhI T14A & PUT21 with replacement of threonine codon 14 of odhI by an alanine codon & This study \\
\hline PUT21odhI T15A & $\begin{array}{l}\text { PUT21 with replacement of threonine codon } 15 \text { of chromosomal odhI } \\
\text { by an alanine codon }\end{array}$ & This study \\
\hline PUT21proB ${ }^{\mathrm{GTG}}$ & $\begin{array}{l}\text { PUT21 with replacement of translational start codon ATG of chromosomal } \\
\text { proB by GTG }\end{array}$ & This study \\
\hline PUT21proB $^{\text {TTG }}$ & $\begin{array}{l}\text { PUT21 with replacement of translational start codon ATG of chromosomal } \\
\text { proB by TTG }\end{array}$ & This study \\
\hline NA2 & $\begin{array}{l}\text { PUT21odh } A^{\mathrm{TTG}} \text { with replacement of threonine codon } 15 \text { of chromosomal } o d h I \text { by } \\
\text { an alanine codon }\end{array}$ & This study \\
\hline NA3 & $\begin{array}{l}\text { NA2, but carrying plasmid pVWEx1-speC-gapA-argF } F_{21} \text { instead of } \\
\text { pVWEx1-speC- } \arg F_{21}\end{array}$ & This study \\
\hline NA4 & $\begin{array}{l}\text { NA2, but carrying plasmid pVWEx1-speC-gapA-pyc-argF } F_{21} \text { instead of pVWEx1- } \\
\text { speC-argF } F_{21}\end{array}$ & This study \\
\hline NA5 & $\begin{array}{l}\text { NA2, but carrying plasmid pVWEx1-speC-gapA-pyc-arg } B^{\mathrm{A} 49 \mathrm{~V} / \mathrm{M} 54 \mathrm{~V}}-\arg F_{21} \text { instead } \\
\text { of pVWEx1-speC-argF } F_{21}\end{array}$ & This study \\
\hline NA6 & NA5 with chromosomal deletion of snaA & This study \\
\hline NA7 & NA6 with chromosomal deletion of $\operatorname{cgm} R$ & This study \\
\hline NA8 & $\begin{array}{l}\text { NA7 with replacement of translational start codon ATG of chromosomal } \\
\text { proB by TTG }\end{array}$ & This study \\
\hline \multicolumn{3}{|l|}{ Plasmids } \\
\hline $\mathrm{pEKEx} 3$ & Spec $^{\mathrm{R}}$; Ptac, lacIq; pBL1, oriVC.g., oriVE.c. & {$[44]$} \\
\hline pEKEx3-gapA & $\mathrm{SpeC}^{\mathrm{R}}, \mathrm{pEKEx} 3$ overexpressing gapA from C. glutamicum ATCC13032 & {$[30]$} \\
\hline pEKEx3-pyc & $\mathrm{SpeC}^{\mathrm{R}}, \mathrm{pEKEx} 3$ overexpressing $p y c$ from C. glutamicum ATCC13032 & {$[27]$} \\
\hline $\mathrm{pEKEx3- \operatorname {arg } B}$ A49V/M54V & $\mathrm{SpeC}^{\mathrm{R}}, \mathrm{pEKEx} 3$ overexpressing $\arg B^{\mathrm{A} 49 \mathrm{~V} / \mathrm{M} 54 \mathrm{~V}}$ from C. glutamicum ATCC13032 & {$[36]$} \\
\hline pVWEx1-speC-argF $F_{21}$ & $\begin{array}{l}\mathrm{Kan}^{\mathrm{R}} \text {, plasmid-based overexpressing speC from } E \text {. coli MG1655 and leaky } \\
\text { expression of } \arg F \text { in pVWEx1 }\end{array}$ & {$[4]$} \\
\hline pK19mobsacBodhA $A^{\mathrm{TTG}}$ & $\begin{array}{l}\mathrm{Kan}^{\mathrm{R}} ; \text { mobilizable vector for the replacement of start codon of } o d h A \text { from GTG to } \\
\text { TTG }\end{array}$ & This study \\
\hline pK19mobsacBodhI $I^{\mathrm{T} 14 \mathrm{~A}}$ & $\mathrm{Kan}^{\mathrm{R}}$; mobilizable vector for the replacement of threonine 14 in $o d h I$ by alanine & This study \\
\hline
\end{tabular}


Table 3. Cont.

\begin{tabular}{|c|c|c|}
\hline Name & Relevant Genotype/Information & Refs. \\
\hline \multicolumn{3}{|l|}{$\underline{\text { Strains }}$} \\
\hline $\begin{array}{c}\text { pVWEx1-speC-gapA-pyc- } \\
\operatorname{argB}^{\mathrm{A49V} / \mathrm{M} 54 \mathrm{~V}}-\arg F_{21}\end{array}$ & $\begin{array}{l}\mathrm{Kan}^{\mathrm{R}}, \mathrm{Kan}^{\mathrm{R}} \text {, plasmid-based overexpressing } \arg B^{\mathrm{A} 49 \mathrm{~V} / \mathrm{M} 54 \mathrm{~V}}, p y c \text { and } g a p A \text { from } \\
\text { C. glutamicum, speC from E. coli MG1655 and leaky expression of } \arg F\end{array}$ & This study \\
\hline pK19mobsacB & $\begin{array}{l}\text { Kan }{ }^{\mathrm{R}} \text {; mobilizable } E \text {. coli vector for the construction of deletion mutants in } \\
\text { C. glutamicum (oriVE.c., PT7, lacI) }\end{array}$ & {$[45]$} \\
\hline pK19mobsacBodhA ${ }^{\mathrm{TTG}}$ & $\begin{array}{l}\operatorname{Kan}^{\mathrm{R}} \text {; mobilizable vector for the replacement of start codon of } o d h A \text { from GTG to } \\
\text { TTG }\end{array}$ & This study \\
\hline pK19mobsacBodhI ${ }^{\mathrm{T} 14 \mathrm{~A}}$ & $\mathrm{Kan}^{\mathrm{R}}$; mobilizable vector for the replacement of threonine 14 in $o d h I$ by alanine & This study \\
\hline pK19mobsacBodhI ${ }^{\mathrm{T} 15 \mathrm{~A}}$ & $\mathrm{Kan}^{\mathrm{R}}$; mobilizable vector for the replacement of threonine 15 in $o d h I$ by alanine & This study \\
\hline pK19mobsacBproB ${ }^{\mathrm{GTG}}$ & $\begin{array}{l}\text { Kan }{ }^{\mathrm{R}} \text {; mobilizable vector for replacement of native start codon ATG of proB } \\
\text { by GTG }\end{array}$ & This study \\
\hline pK19mobsacBproB ${ }^{\mathrm{TTG}}$ & $\begin{array}{l}\text { Kan }{ }^{\mathrm{R}} \text {; mobilizable vector for replacement of native start codon ATG of proB } \\
\text { by TTG }\end{array}$ & This study \\
\hline pK19mobsacB $\Delta$ snaA & $\mathrm{Kan}^{\mathrm{R}}$; mobilizable vector for deletion of $\operatorname{snaA}$ & {$[39]$} \\
\hline pK19mobsacB $\Delta$ cgmR & $\mathrm{Kan}^{\mathrm{R}}$; mobilizable vector for deletion of $\operatorname{cgmR}$ & {$[39]$} \\
\hline
\end{tabular}

\subsection{Strain Construction}

The oligonucleotides used as PCR primers in this study are listed in Table 4. Plasmids were constructed in Escherichia coli DH5 $\alpha$ by standard molecular genetic methods and confirmed by DNA sequence analysis. Transformation of $E$. coli was performed using the rubidium chloride method [46] while C. glutamicum was transformed by electroporation as described previously [43]. C. glutamicum deletion mutants were constructed by crossover PCR (or Gibson Assembly) and double homologous recombination using the suicide vector pK19mobsacB [45]. For plasmid-based overexpression, ORFs of corresponding genes were amplified using PCR and ligated into digested plasmid pEKEx3 or pVWEx 1 or cloned by Gibson Assembly [47]. The resulting strains are listed in Table 3.

Table 4. List of Primers.

\begin{tabular}{cl}
\hline Primer names & Sequence $\left(\mathbf{5}^{\prime} \mathbf{- 3}\right.$ ') \\
\hline odhI141 & CGAATCCATTCACCTGC \\
odhI142 & ACTGAGGTGGCCTCGACCTG \\
odhI143 & CAGGTCGAGGCCACCTCAGT \\
odhI144 & GCAACCGCACTGTTTG \\
odhI152 & ACTGAGGCGGTCTCGACCTG \\
odhI153 & CAGGTCGAGACCGCCTCAGT \\
odhA1 & CCTGATGGTTTCAACCATCAAGTC \\
odhA2 & AGTACTAGCGCTGCTCAAGGCAGG \\
odhA3 & CTGCCTTGAGCAGCGCTAGTAC \\
odhA4 & CCATGGCGTAGCCAATGATG \\
gtg1 & AGCAGTTGGCTACCTGG \\
$\operatorname{gtg} 2$ & CACCGGCGCCACTTGGGTTG \\
gtg3 & CAACCCAAGTGGCGCCGGTG \\
gtg4 & GGCAAAAGAACGTCCCC \\
\hline
\end{tabular}


Table 4. Cont.

\begin{tabular}{|c|c|}
\hline Primer names & Sequence $\left(5^{\prime}-3^{\prime}\right)$ \\
\hline $\operatorname{ttg} 2$ & ACCGGCGCCAATTGGGTTGG \\
\hline $\operatorname{ttg} 3$ & CCAACCCAATTGGCGCCGGT \\
\hline gapA-cgl-fw & AAGGAGATATAGATATGACCATTCGTGTTGGTATTAAC \\
\hline gapA-cgl-rv & TTAGAGCTTGGAAGCTACGAGCTC \\
\hline ACBF-gapA 1 & $\begin{array}{l}\text { TTGTACGGTTATGTGTTGAAGTAAGGATCCGAAAGGAGGCCCTTCAGATGACC } \\
\text { ATTCGTGTTGGTATTA }\end{array}$ \\
\hline ACBF-gapA2 & ATCTGAAGGGCCTCCTTTCACATGTTTAGAGCTTGGAAGCTACGAG \\
\hline ACBF-gapA3 & AGTGAATTCGAGCTCGGTACCCGGGCATATGTTAGAGCTTGGAAGCTACGAG \\
\hline ACBF-pyc 1 & $\begin{array}{l}\text { CTCGTAGCTTCCAAGCTCTAAACATGTGAAAGGAGGCCCTTCAGATGTCGACT } \\
\text { CACACATCTTC }\end{array}$ \\
\hline ACBF-pyc2 & GGCCTCCTTTCGCGGCCGCTTAGGAAACGACGACGATCA \\
\hline ACBF-pyc3 & AGTGAATTCGAGCTCGGTACCCGGGCATATGTTAGGAAACGACGACGATCA \\
\hline $\mathrm{ACBF}-\arg B 1$ & TGATCGTCGTCGTTTCCTAAGCGGCCGCGAAAGGAGGCCCTTCAGTTG \\
\hline $\mathrm{ACBF}-\arg B 2$ & AGTGAATTCGAGCTCGGTACCCGGGCATATGTTACAGTTCCCCATCCTTGTC \\
\hline
\end{tabular}

\subsection{Flux Balance Analysis}

The flux balance analysis was carried out using Matlab (Mathworks, Natick, MA, USA) and the COBRA 2.0 toolbox, together with the GNU Linear Programming Kit [48]. The genome scale model of C. glutamicum was taken from [49]. The following changes were made to the original model: (1) putrescine secretion capability was added, (2) L-arginine interconversion was constrained to zero, (3) glycerol and lactate uptake was added, (4) cofactor of the glycerol-3-phosphate dehydrogenase was changed to NAD, (5) the acetate kinase reaction was set reversible to allow acetate consumption, and (6) $\mathrm{NAD}(\mathrm{P}) \mathrm{H}$ futile reactions were added for capacity estimations (see also supplementary Table S2). All flux units are in mmol ( $\mathrm{gCDW} \mathrm{h})^{-1}$, except for the flux into biomass formation, which has units of $\mathrm{h}^{-1}$. The substrate uptake was varied between 0 and $4 \mathrm{mmol}(\mathrm{gCDW} \mathrm{h})^{-1}$. Non growth associated ATP demand was set zero. All other fluxes were left unconstrained, if not otherwise mentioned. Finally, the identification of a particular flux distribution was formulated as a constrained linear programming problem, in which the objective function was maximized. The objective function was (1) biomass formation, (2) putrescine secretion, or (3) cofactor production (ATP, NADH, NADPH). Shadow prices were determined using COBRA.

\subsection{Enzyme Assays}

2-oxoglutarate dehydrogenase activity was assayed as described previously [50]. Only fresh extracts were used, prepared in $0.1 \mathrm{M}$ TES [ $N$-tris (hydroxymethyl) methyl-2-aminoethanesulfonic acid] ( $\mathrm{pH} 7.2), 10 \mathrm{mM} \mathrm{MgCl} 2,3 \mathrm{mM}$ cysteine, 10 vol-\% glycerol. The assay mixture $(1 \mathrm{~mL})$ was $50 \mathrm{mM}$ TES (pH 7.7), $10 \mathrm{mM} \mathrm{MgCl}$, $3 \mathrm{mM}$ cysteine, $2 \mathrm{mM}$ NAD, $0.9 \mathrm{mM}$ thiamine diphosphate, $0.05 \mathrm{mM}$ chlorpromazine, and $2.5 \mathrm{mM}$ 2-oxoglutarate. The reaction was performed at $30{ }^{\circ} \mathrm{C}$, started with 0.2 $\mathrm{mM}$ CoA, and extinction followed at $340 \mathrm{~nm}$. 
Glyceraldehyde 3-phosphate dehydrogenase activity was measured as previously reported [51]. The assay contained $1 \mathrm{mM} \mathrm{NAD}^{+}, 50 \mathrm{mM} \mathrm{Na}_{2} \mathrm{HPO}_{4}, 0.2 \mathrm{mM}$ EDTA, and $0.5 \mathrm{mM}$ glyceraldehyde

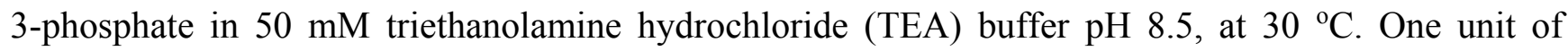
enzyme activity corresponds to $1 \mu \mathrm{mol}$ NADH formed per minute.

$\mathrm{N}$-Acetylglutamate 5-phosphotransferase activity was performed based on previous report [52]. The incubation mixture consisted of: $400 \mathrm{mM} \mathrm{NH} 3 \mathrm{OH}-\mathrm{HCl}, 400 \mathrm{mM}$ Tris (base), $40 \mathrm{mM}$ $\mathrm{N}$-acetyl-L-glutamate, $20 \mathrm{mM} \mathrm{MgCl} 2,10 \mathrm{mM}$ ATP and up to 0.03 unit of enzyme in a final volume of $1.0 \mathrm{~mL}$. The $\mathrm{pH}$ of the incubation mixture containing all components except N-acetyl-L-glutamate and enzyme was adjusted to 7.2 with $\mathrm{NaOH}$ at $25{ }^{\circ} \mathrm{C}$. The reaction was started by the addition of $\mathrm{N}$-acetyl-L-glutamate. After incubation at $30{ }^{\circ} \mathrm{C}$ for $10-20 \mathrm{~min}$ the reaction was terminated by the addition of $1.0 \mathrm{~mL}$ of a solution containing $5 \%(\mathrm{w} / \mathrm{v}) \mathrm{FeCl}_{3}$ hexahydrate, $8 \%(\mathrm{w} / \mathrm{v})$ trichloroacetic acid, and $0.3 \mathrm{M} \mathrm{HCl}$. When necessary the precipitate was removed by centrifugation. The volume of all reagents was halved for the assay of purified enzyme. The absorbance of the resulting hydroxamate- $\mathrm{Fe}^{3+}$ complex was measured at $540 \mathrm{~nm}$. One unit of $\mathrm{N}$-acetylglutamate 5-phosphotransferase is defined as the amount of enzyme which catalyzes the formation of of $1 \mu$ mol of product per min under assay conditions.

\subsection{Quantification of Putrescine}

For quantification of extracellular putrescine, aliquots of the culture were withdrawn, the optical density (OD 600$)$ was measured and cells were removed by centrifugation $(13,000 \times \mathrm{g}, 10 \mathrm{~min})$. The supernatant was analyzed using a high-pressure liquid chromatography system (HPLC, 1200 series, Agilent Technologies Deutschland GmbH, Böblingen, Germany).

Putrescine were determined by precolumn derivatization with ortho-phthaldialdehyde and separated

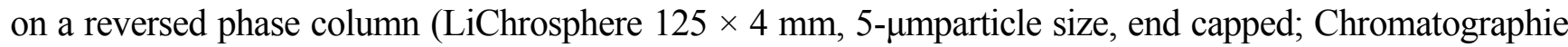
Service $\mathrm{GmbH}$, Langerwehe, Germany). The respective fluorescent isoindole derivatives were detected by excitation at $330 \mathrm{~nm}$ (emission at $450 \mathrm{~nm}$ ). For elution gradients of $2.5 \mathrm{~g} \cdot \mathrm{L}^{-1}$ sodium acetate, $\mathrm{pH} 6.0$, as the polar phase and methanol as the nonpolar phase were used. Diaminohexane was used as an internal standard [11].

\section{Conclusions}

Taken together, the previously available putrescine producing strain C. glutamicum PUT21 was improved by combinatorial engineering guided by flux balance analysis. In shake flask cultivations $58.1 \pm 0.2 \mathrm{mM}$ or about $5.1 \mathrm{~g} \cdot \mathrm{L}^{-1}$ putrescine accumulated and $C$. glutamicum NA6 produced putrescine with $56 \%$ of the maximal product yield (i.e., $0.26 \mathrm{~g} \cdot \mathrm{g}^{-1}$ ). An overall volumetric productivity of $0.21 \mathrm{~g} \cdot \mathrm{L}^{-1} \cdot \mathrm{h}^{-1}$, an overall specific productivity of $0.045 \mathrm{~g} \cdot \mathrm{g}^{-1} \cdot \mathrm{h}^{-1}$, and a biomass yield of $0.23 \mathrm{~g} \cdot \mathrm{g}^{-1}$ were observed (Table 2). Three of the chosen metabolic engineering targets led to the largest improvements: feedback alleviated $\mathrm{N}$-acetylglutamate kinase, reduced ODHC activity and preventing $\mathrm{N}$-acetylation of putrescine. 


\section{Acknowledgments}

Anh Q. D. Nguyen is a fellow of the CLIB2021 graduate cluster at Bielefeld University. The authors acknowledge support of the publication fee by Deutsche Forschungsgemeinschaft and the Open Access Publication Funds of Bielefeld University.

\section{Author Contributions}

Volker F. Wendisch, Anh Q. D. Nguyen and Jens Schneider designed the experiments. Jens Schneider performed flux balance analysis, Anh Q. D. Nguyen and Gajendar Komati Reddy conducted metabolic engineering experiments, Anh Q. D. Nguyen performed all cultivations and their characterization. Anh Q. D. Nguyen, Gajendar Komati Reddy and Jens Schneider analyzed the results, Anh Q. D. Nguyen drafted the manuscript. Volker F. Wendisch reviewed and revised the manuscript. All authors read and approved the final manuscript.

\section{Conflicts of Interest}

The authors do not declare competing interests.

\section{References}

1. Schaffer, S.; Haas, T. Biocatalytic and fermentative production of alpha,omega-bifunctional polymer precursors. Org. Process. Res. Dev. 2014, 18, 752-766. Available online: http://dx.doi.org/ 10.1021/op5000418 (accessed on 11 February 2015).

2. Rydz, J.; Sikorska, W.; Kyulavska, M.; Christova, D. Polyester-based (bio)degradable polymers as environmentally friendly materials for sustainable development. Int. J. Mol. Sci. 2014, 16, 564-596.

3. Kroschwitz, J.I.; Seidel, A. Kirk-Othmer Encyclopedia of Chemical Technology, 5th ed.; Wiley-Interscienc: Hoboken, NJ, USA, 2004.

4. Schneider, J.; Eberhardt, D.; Wendisch, V.F. Improving putrescine production by Corynebacterium glutamicum by fine-tuning ornithine transcarbamoylase activity using a plasmid addiction system. Appl. Microbiol. Biotechnol. 2012, 95, 169-178.

5. Schneider, J.; Wendisch, V.F. Putrescine production by engineered Corynebacterium glutamicum. Appl. Microbiol. Biotechnol. 2010, 88, 859-868.

6. Qian, Z.G.; Xia, X.X.; Lee, S.Y. Metabolic engineering of Escherichia coli for the production of putrescine: A four carbon diamine. Biotechnol. Bioeng. 2009, 104, 651-662.

7. Meiswinkel, T.M.; Rittmann, D.; Lindner, S.N.; Wendisch, V.F. Crude glycerol-based production of amino acids and putrescine by Corynebacterium glutamicum. Bioresour. Technol. 2013, 145, 254-258.

8. Meiswinkel, T.M.; Gopinath, V.; Lindner, S.N.; Nampoothiri, K.M.; Wendisch, V.F. Accelerated pentose utilization by Corynebacterium glutamicum for accelerated production of lysine, glutamate, ornithine and putrescine. Microb. Biotechnol. 2013, 6, 131-140. 
9. Uhde, A.; Youn, J.W.; Maeda, T.; Clermont, L.; Matano, C.; Kramer, R.; Wendisch, V.F.; Seibold, G.M.; Marin, K. Glucosamine as carbon source for amino acid-producing Corynebacterium glutamicum. Appl. Microbiol. Biotechnol. 2013, 97, 1679-1687.

10. Meiswinkel, T.M.; Lindner, S.N.; Wendisch, V.F. Thick juice-based production of amino acids and putrescine by Corynebacterium glutamicum. J. Biotechnol. Biomater. 2014, doi:10.4172/ 2155-952X.1000167.

11. Peters-Wendisch, P.; Gotker, S.; Heider, S.A.; Komati Reddy, G.; Nguyen, A.Q.; Stansen, K.C.; Wendisch, V.F. Engineering biotin prototrophic Corynebacterium glutamicum strains for amino acid, diamine and carotenoid production. J. Biotechnol. 2014, 192, 346-354.

12. Shinfuku, Y.; Sorpitiporn, N.; Sono, M.; Furusawa, C.; Hirasawa, T.; Shimizu, H. Development and experimental verification of a genome-scale metabolic model for Corynebacterium glutamicum. Microb. Cell. Fact. 2009, doi:10.1186/1475-2859-8-43.

13. Blombach, B.; Riester, T.; Wieschalka, S.; Ziert, C.; Youn, J.W.; Wendisch, V.F.; Eikmanns, B.J. Corynebacterium glutamicum tailored for efficient isobutanol production. Appl. Environ. Microbiol. 2011, 77, 3300-3310.

14. Rittmann, D.; Lindner, S.N.; Wendisch, V.F. Engineering of a glycerol utilization pathway for amino acid production by Corynebacterium glutamicum. Appl. Environ. Microbiol. 2008, 74, 6216-6222.

15. Hadiati, A.; Krahn, I.; Lindner, S.N.; Wendisch, V.F. Engineering of Corynebacterium glutamicum for growth and production of L-ornithine, L-lysine, and lycopene from hexuronic acids. Bioresour. Bioprocess. 2014, doi:10.1186/s40643-014-0025-5.

16. Usuda, Y.; Tujimoto, N.; Abe, C.; Asakura, Y.; Kimura, E.; Kawahara, Y.; Kurahashi, O.; Matsui, H. Molecular cloning of the Corynebacterium glutamicum ("Brevibacterium lactofermentum" AJ12036) odhA gene encoding a novel type of 2-oxoglutarate dehydrogenase. Microbiology 1996, 142, 3347-3354.

17. Schwinde, J.W.; Hertz, P.F.; Sahm, H.; Eikmanns, B.J.; Guyonvarch, A. Lipoamide dehydrogenase from Corynebacterium glutamicum: Molecular and physiological analysis of the lpd gene and characterization of the enzyme. Microbiology 2001, 147, 2223-2231.

18. Niebisch, A.; Kabus, A.; Schultz, C.; Weil, B.; Bott, M. Corynebacterial protein kinase G controls 2-oxoglutarate dehydrogenase activity via the phosphorylation status of the OdhI protein. J. Biol. Chem. 2006, 281, 12300-12307.

19. Kawahara, Y.; Takahashi-Fuke, K.; Shimizu, E.; Nakamatsu, T.; Nakamori, S. Relationship between the glutamate production and the activity of 2-oxoglutarate dehydrogenase in Brevibacterium lactofermentum. Biosci. Biotechnol. Biochem. 1997, 61, 1109-1112. Available online: http://dx.doi.org/10.1271/bbb.61.1109 (accessed on 24 July 2014).

20. Asakura, Y.; Kimura, E.; Usuda, Y.; Kawahara, Y.; Matsui, K.; Osumi, T.; Nakamatsu, T. Altered metabolic flux due to deletion of odhA causes L-glutamate overproduction in Corynebacterium glutamicum. Appl. Environ. Microbiol. 2006, 73, 1308-1319.

21. Raasch, K.; Bocola, M.; Labahn, J.; Leitner, A.; Eggeling, L.; Bott, M. Interaction of 2oxoglutarate dehydrogenase OdhA with its inhibitor OdhI in Corynebacterium glutamicum: Mutants and a model. J. Biotechnol. 2014, 191, 99-105. 
22. Krawczyk, S.; Raasch, K.; Schultz, C.; Hoffelder, M.; Eggeling, L.; Bott, M. The FHA domain of OdhI interacts with the carboxyterminal 2-oxoglutarate dehydrogenase domain of OdhA in Corynebacterium glutamicum. FEBS Lett. 2010, 584, 1463-1468.

23. Schultz, C.; Niebisch, A.; Schwaiger, A.; Viets, U.; Metzger, S.; Bramkamp, M.; Bott, M. Genetic and biochemical analysis of the serine/threonine protein kinases PknA, PknB, PknG and PknL of Corynebacterium glutamicum: Evidence for non-essentiality and for phosphorylation of OdhI and FtsZ by multiple kinases. Mol. Microbiol. 2009, 74, 724-741.

24. Schultz, C.; Niebisch, A.; Gebel, L.; Bott, M. Glutamate production by Corynebacterium glutamicum: Dependence on the oxoglutarate dehydrogenase inhibitor protein OdhI and protein kinase PknG. Appl. Microbiol. Biotechnol. 2007, 76, 691-700.

25. Yukawa, H.; Inui, M.; Vertes, A.A. Genomes and genome-level engineering of amino acid-producing bacteria. In Amino Acid Biosynthesis-Pathways, Regulation and Metabolic Engineering; Wendisch, V.F., Ed.; Springer: Heidelberg, Germany, 2007; Volume 5, pp. 349-401, doi:10.1007/ 7171_2006_066.

26. Peters-Wendisch, P.G.; Wendisch, V.F.; Paul, S.; Eikmanns, B.J.; Sahm, H. Pyruvate carboxylase as an anaplerotic enzyme in Corynebacterium glutamicum. Microbiology 1997, 143, 1095-1103.

27. Peters-Wendisch, P.G.; Schiel, B.; Wendisch, V.F.; Katsoulidis, E.; Mockel, B.; Sahm, H.; Eikmanns, B.J. Pyruvate carboxylase is a major bottleneck for glutamate and lysine production by Corynebacterium glutamicum. J. Mol. Microbiol. Biotechnol. 2001, 3, 295-300.

28. Jojima, T.; Fujii, M.; Mori, E.; Inui, M.; Yukawa, H. Engineering of sugar metabolism of Corynebacterium glutamicum for production of amino acid L-alanine under oxygen deprivation. Appl. Microbiol. Biotechnol. 2010, 87, 159-165.

29. Shirai, T.; Fujimura, K.; Furusawa, C.; Nagahisa, K.; Shioya, S.; Shimizu, H. Study on roles of anaplerotic pathways in glutamate overproduction of Corynebacterium glutamicum by metabolic flux analysis. Microb. Cell. Fact. 2007, doi:10.1186/1475-2859-6-19.

30. Siedler, S.; Lindner, S.N.; Bringer, S.; Wendisch, V.F.; Bott, M. Reductive whole-cell biotransformation with Corynebacterium glutamicum: Improvement of NADPH generation from glucose by a cyclized pentose phosphate pathway using pfkA and gapA deletion mutants. Appl. Microbiol. Biotechnol. 2013, 97, 143-152.

31. Jiang, L.Y.; Chen, S.G.; Zhang, Y.Y.; Liu, J.Z. Metabolic evolution of Corynebacterium glutamicum for increased production of L-ornithine. BMC Biotechnol. 2013, doi:10.1186/1472-6750-13-47.

32. Kim, S.Y.; Lee, J.; Lee, S.Y. Metabolic engineering of Corynebacterium glutamicum for the production of L-ornithine. Biotechnol. Bioeng. 2015, 112, 416-421.

33. Lee, S.Y.; Shin, H.S.; Park, J.S.; Kim, Y.H.; Min, J. Proline reduces the binding of transcriptional regulator $\mathrm{ArgR}$ to upstream of $\operatorname{argB}$ in Corynebacterium glutamicum. Appl. Microbiol. Biotechnol. 2010, 86, 235-242.

34. Ronsch, H.; Kramer, R.; Morbach, S. Impact of osmotic stress on volume regulation, cytoplasmic solute composition and lysine production in Corynebacterium glutamicum MH20-22B. J. Biotechnol. 2003, 104, 87-97.

35. Weinand, M.; Kramer, R.; Morbach, S. Characterization of compatible solute transporter multiplicity in Corynebacterium glutamicum. Appl. Microbiol. Biotechnol. 2007, 76, 701-708. 
36. Schneider, J.; Niermann, K.; Wendisch, V.F. Production of the amino acids L-glutamate, L-lysine, L-ornithine and L-arginine from arabinose by recombinant Corynebacterium glutamicum. J. Biotechnol. 2011, 154, 191-198.

37. Xu, M.; Rao, Z.; Dou, W.; Jin, J.; Xu, Z. Site-directed mutagenesis studies on the L-arginine-binding sites of feedback inhibition in N-acetyl-L-glutamate kinase (NAGK) from Corynebacterium glutamicum. Curr. Microbiol. 2012, 64, 164-172.

38. Schendzielorz, G.; Dippong, M.; Grunberger, A.; Kohlheyer, D.; Yoshida, A.; Binder, S.; Nishiyama, C.; Nishiyama, M.; Bott, M.; Eggeling, L. Taking control over control: Use of product sensing in single cells to remove flux control at key enzymes in biosynthesis pathways. $A C S$ Synth. Biol. 2014, 3, 21-29.

39. Nguyen, A.Q.; Schneider, J.; Wendisch, V.F. Elimination of polyamine N-acetylation and regulatory engineering improved putrescine production by Corynebacterium glutamicum. J. Biotechnol. 2015, 201, 75-85.

40. Itou, H.; Watanabe, N.; Yao, M.; Shirakihara, Y.; Tanaka, I. Crystal structures of the multidrug binding repressor Corynebacterium glutamicum CgmR in complex with inducers and with an operator. J. Mol. Biol. 2010, 403, 174-184.

41. Kind, S.; Kreye, S.; Wittmann, C. Metabolic engineering of cellular transport for overproduction of the platform chemical 1,5-diaminopentane in Corynebacterium glutamicum. Metab. Eng. 2011, 13, 617-627.

42. Kind, S.; Jeong, W.K.; Schroder, H.; Zelder, O.; Wittmann, C. Identification and elimination of the competing $\mathrm{N}$-acetyldiaminopentane pathway for improved production of diaminopentane by Corynebacterium glutamicum. Appl. Environ. Microbiol. 2010, 76, 5175-5180,

43. Eggeling, L.; Bott, M. Handbook of Corynebacterium Glutamicum; CRC Press: Boca Raton, FL, USA, 2005.

44. Stansen, C.; Uy, D.; Delaunay, S.; Eggeling, L.; Goergen, J.L.; Wendisch, V.F. Characterization of a Corynebacterium glutamicum lactate utilization operon induced during temperature-triggered glutamate production. Appl. Environ. Microbiol. 2005, 71, 5920-5928.

45. Schäfer, A.; Tauch, A.; Jäger, W.; Kalinowski, J.; Thierbach, G.; Puhler, A. Small mobilizable multi-purpose cloning vectors derived from the Escherichia coli plasmids pK18 and pK19: selection of defined deletions in the chromosome of Corynebacterium glutamicum. Gene 1994, 145, 69-73.

46. Hanahan, D. Studies on transformation of Escherichia coli with plasmids. J. Mol. Biol. 1983, 166, $557-580$.

47. Gibson, D.G.; Young, L.; Chuang, R.Y.; Venter, J.C.; Hutchison, C.A., 3rd; Smith, H.O. Enzymatic assembly of DNA molecules up to several hundred kilobases. Nat. Methods 2009, 6, 343-345.

48. Schellenberger, J.; Que, R.; Fleming, R.M.; Thiele, I.; Orth, J.D.; Feist, A.M.; Zielinski, D.C.; Bordbar, A.; Lewis, N.E.; Rahmanian, S., et al. Quantitative prediction of cellular metabolism with constraint-based models: the COBRA Toolbox v2.0. Nat. Protoc. 2011, 6, 1290-1307.

49. Shen, L.; Haufe, J.; Patel, M.K. Product overview and market projection of emerging bio-based plastics (Utrecht University). 2009. Available online: http://en.european-bioplastics.org (accessed on 9 February 2015). 
50. Hoffelder, M.; Raasch, K.; van Ooyen, J.; Eggeling, L. The E2 domain of OdhA of Corynebacterium glutamicum has succinyltransferase activity dependent on lipoyl residues of the acetyltransferase AceF. J. Bacteriol. 2010, 192, 5203-5211.

51. Omumasaba, C.A.; Okai, N.; Inui, M.; Yukawa, H. Corynebacterium glutamicum glyceraldehyde3-phosphate dehydrogenase isoforms with opposite, ATP-dependent regulation. J. Mol. Microbiol. Biotechnol. 2004, 8, 91-103.

52. Haas, D.; Leisinger, T. N-acetylglutamate 5-phosphotransferase of Pseudomonas aeruginosa. Purification and ligand-directed association-dissociation. Eur. J. Biochem. 1975, 52, 365-375.

(C) 2015 by the authors; licensee MDPI, Basel, Switzerland. This article is an open access article distributed under the terms and conditions of the Creative Commons Attribution license (http://creativecommons.org/licenses/by/4.0/). 